\title{
Miniature Twist/Rotation Fabry Perot Sensor Based on a Four-Core Fiber ${ }^{+}$
}

\author{
Vedran Budinski * and Denis Donlagic \\ Laboratory for Electro-Optic Sensor Systems, UM FERI, Koroška cesta 46, 2000 Maribor, Slovenia; \\ denis.donlagic@um.si \\ * Correspondence: vedran.budinski@um.si; Tel.: +386-2-220-7107 \\ † Presented at the Eurosensors 2018 Conference, Graz, Austria, 9-12 September 2018. \\ Published: 11 December 2018
}

\begin{abstract}
This paper presents a miniature Fabry Perot twist/rotation sensor. The presented sensor consists of a single lead-in multicore fiber, which has four eccentrically positioned cores, a special asymmetrical microstructure, similar to a truncated cylinder, and an inline semi reflective mirror, all packed in a glass capillary housing. The perpendicular cut lead-in multicore fiber and the inline semi reflective mirror form four Fabry-Perot cavities. The optical path length of each Fabry-Perot interferometer is defined by the distance between mirrors, refractive index and twist/rotation angle of the microstructure in relation to the core positions in the lead in multicore fiber. Optical paths of Fabry-Perot Interferometers are modulated by a structure's twist/rotation, change of structure length, or change of temperature. Each of these parameters modulate the optical path length of the individual interferometers in their own separate fashion, thus allowing independent measurements of twist/rotation, length/strain and temperature.
\end{abstract}

Keywords: optical fiber sensor; rotation sensor; multi core fiber; Fabry-Perot

\section{Introduction}

Implementation of fiber optic twist/rotation sensors proved to be a robust and efficient technology in several industrial fields where active monitoring of twist/rotation parameters is essential. However, a greater breakthrough of this technology is restrained due to current fiber optic sensor twist/rotation solutions, employing different configurations of specialty fibers, which either depend on in-line configurations or a combination of specialized gratings [1]. In-line configuration is often limiting in various applications, as it requires physical access to the measurement location from two opposite directions using two lead fibers. Limited fiber bend diameter thus limits the minimum size of the device to impractical dimensions in the cases of applications requiring small sizes or volumes. Sensing systems that depend on (specialized) gratings can be configured for operating in simpler and more compact back-reflection modes, however, like in-line configurations, they employ complex and cost-prohibitive signal interrogation methods which also experience limited temperature stability. For the purpose of miniaturization and simplification, specialty multicore fibers with Fabry-Perot interferometry are presenting themselves as a technology for constructing micro components/structures for exploitation in new twist/rotation sensor designs. Implementation of Fabry-Perot interferometers is already a proven technique for production of compact pressure sensors [2,3], strain sensors [4], temperature sensors [5] and other sensors for measuring a variety of physical parameters [6,7]. Incorporating a Fabry-Perot interferometer in a twist/rotation fiber sensor presents a unique challenge. Typically, applied pressure, strain, temperature, etc. affect the change in distance between the mirrors of the Fabry-Perot Interferometer. On the other hand, twist/rotation applied to the sensor produces no linear displacement, which, in a homogeneous medium, i.e., glass 
or air, does not cause a variation in the length of the Fabry-Perot interferometer, thus no change in the optical path length. To overcome this limitation, we introduce an asymmetric microstructure that modulates path length with the respect to twist/rotation of the sensor structure.

\section{Sensor Design and Operation}

The proposed sensor is comprised of a single lead-in multicore fiber, with four eccentrically positioned cores, a special asymmetrical microstructure, and an inline semi reflective mirror, all packed in a glass capillary housing (Figure 1b). An asymmetrical microstructure presents an angle polished piece of fiber. When the sensor is subjected to twist/rotation, the asymmetrical microstructure, inline semi reflective mirror and multicore fiber, with eccentrically positioned cores (Figure 1a), define four Fabry-Perot Interferometers (FPIs) with rotation-dependent path lengths.

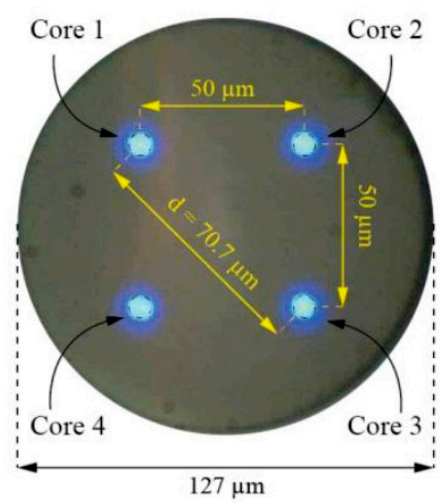

(a)

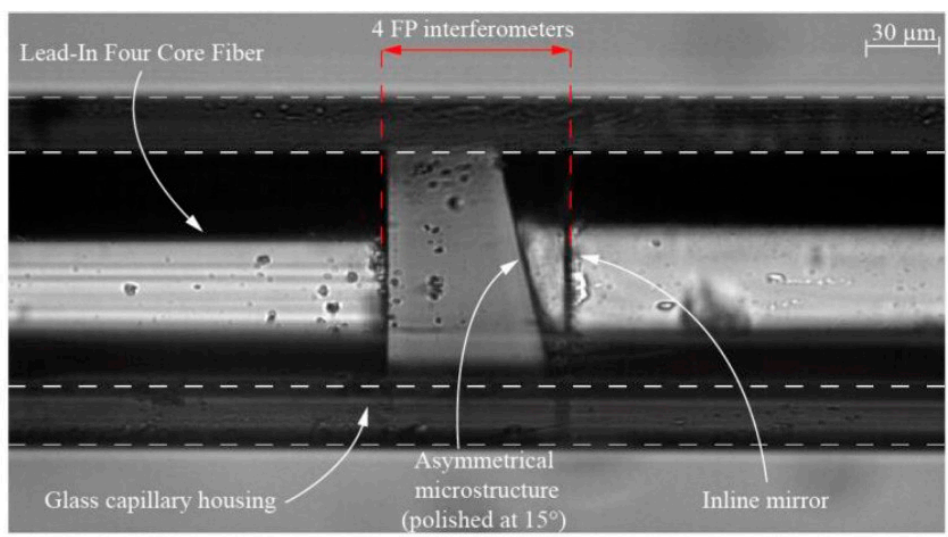

(b)

Figure 1. (a) Cross-section view of the four-core fiber and (b) Sensor assembly.

By measuring the optical path lengths for all four cores, the proposed sensor enables independent measurements of twist/rotation, strain and temperature. In order to determine the twist/rotation angle of the sensor and the rotation direction, variation of the optical paths' lengths for two diametrical FPIs needs to be acquired. Measurements of the optical path length changes for each FPI were obtained by phase-tracking of characteristic components in a Fourier Transform (FT) of the sensor's Back-Reflected Optical Spectrum (BROS). In our experimental characterization we used a National Instrument NI PXIe-4844 spectral interrogator to acquire sensor spectral characteristics (we performed $80 \mathrm{~nm}$ wide sweeps with a $10 \mathrm{~Hz}$ repetition rate). These spectral characteristics were then transferred to a Personal Computer (PC) for processing using a custom-developed code in LabView that relied extensively on FT analysis. Each peak corresponds to individual interferometers' round trip times of flight (i.e., interferometers' optical lengths, when multiplied by one half of the vacuum speed of light). In order to observe the small path-length changes of all four FPIs with high resolution, we further calculated/observed those phases of FT components corresponding to all four FPIs' roundtrip times of flights (lengths), i.e., we observed those phases of the FT components where peaks occurred in the amplitude of the FT of BROS. These phase changes corresponded to the interferometers' optical path length variations $\left(\triangle O P L_{N}\right)$, i.e.,:

$$
\Delta \Phi_{N}=\frac{4 \pi}{\lambda} \Delta O P L N
$$

Thus, the phase change of the two components corresponding to the round-trip-time of flight of the diametrical FPI in the FT of BROS can be correlated to twist/rotation change as:

$$
\sin \Phi=\frac{\lambda\left(\Delta \Phi_{3}-\Delta \Phi_{1}\right)}{4 \pi d \tan \delta_{p}\left(n_{1}-n_{2}\right)}+\sin \Phi_{0}
$$


where $\Delta \Phi_{1}$ and $\Delta \Phi_{3}$ correspond to the phase of the diametrical FPIs in the FT of BROS, $d$ corresponds to the distance between two diametrical fiber cores (Figure 1a), $\delta_{p}$ corresponds to the angle between the longitudinal axis of the fiber and the polished plane of the microstructure (Figure 1b). $n_{1}$ and $n_{2}$ correspond to the refractive indices of air and silica i.e., 1 and 1.444 respectively.

\section{Experimental Results}

The experimental setup is illustrated in Figure 2. A fan-out unit was used to decouple light from a four core fiber to four separate single core fibers. The sensor was fixed between two clamps. For measuring twist/rotation, the second clamp was mounted on a rotational stage with angular resolution of $0.01^{\circ}$. For measuring elongation, the same clamp was attached to a linear stage with a resolution of $1 \mu \mathrm{m}$.

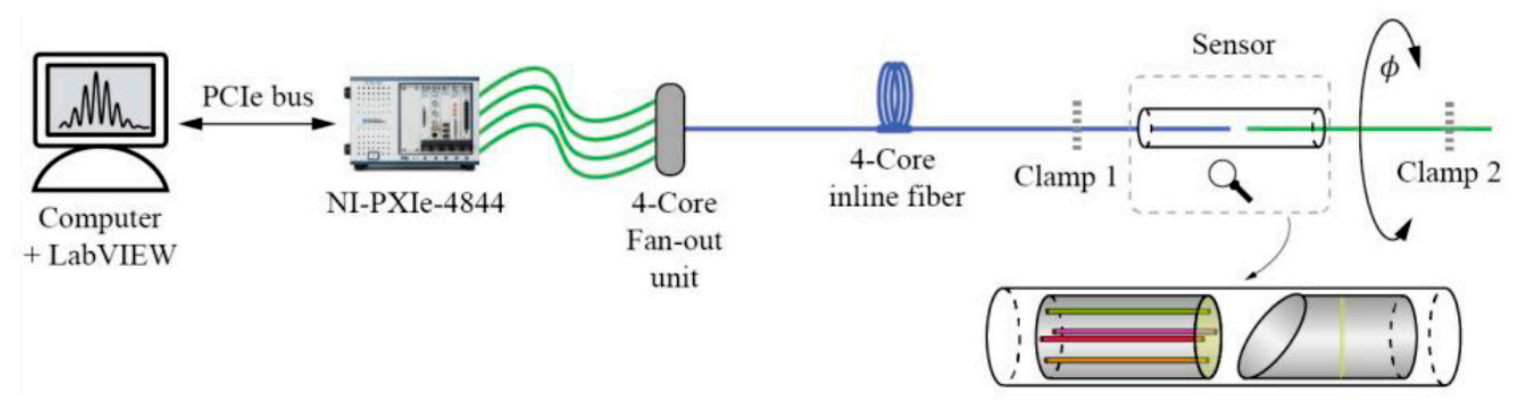

Figure 2. Experimental setup.

In the presented configuration, the sensors' measured ambiguous range was $\pm 90^{\circ}$ (Figure 3a), at a resolution of $0.023^{\circ}$ (Figure $3 b$ ).

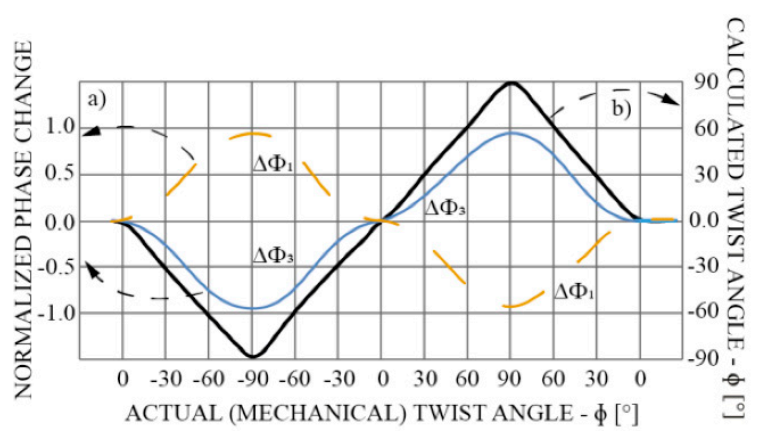

a)

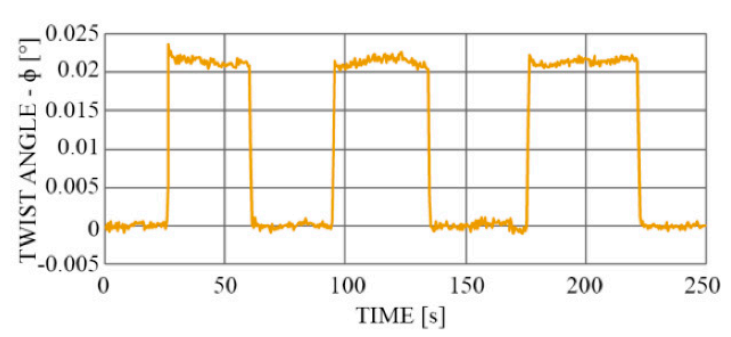

b)

Figure 3. (a) Calculated twist/rotation angle from diametrically opposite FPIs' phases measurement and (b) Minimum resolution measurements when rotating the rotation stage for $0.02^{\circ}$ in the CW direction.

The values of the phases calculated from the spectral characteristics of the FPIs using the DFT algorithm were also used to measure the strain and temperature independently from twist/rotation. The sensor was exposed to an elongation, which extended the latter by $4 \mu \mathrm{m}$ (Figure $4 \mathrm{a}$ ). The sensor was also tested for temperature measurements in the range of $25^{\circ} \mathrm{C}$ to $125^{\circ} \mathrm{C}$ (Figure $4 \mathrm{~b}$ ). 


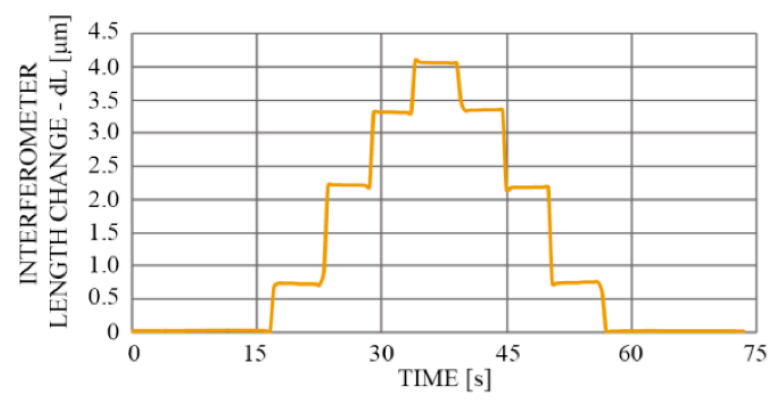

(a)

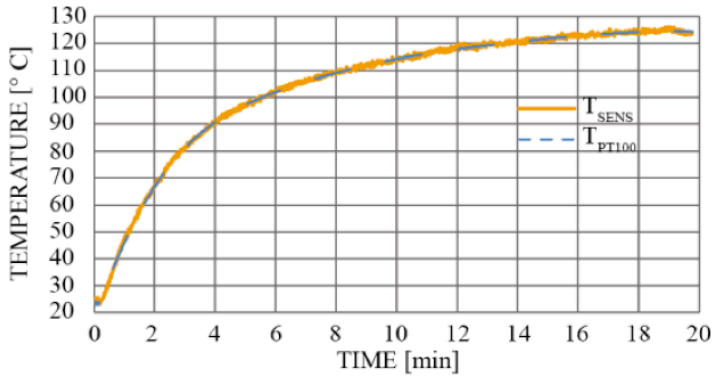

(b)

Figure 4. (a) Sensor elongation test for $4 \mu \mathrm{m}$ and (b) Temperature measurement and comparison with a reference temperature sensor (PT100).

In both cases, the influence of the change in length and temperature on the angle measurement ranged between $0.012^{\circ}$ and $0.013^{\circ}$ (Figure 5a,b).

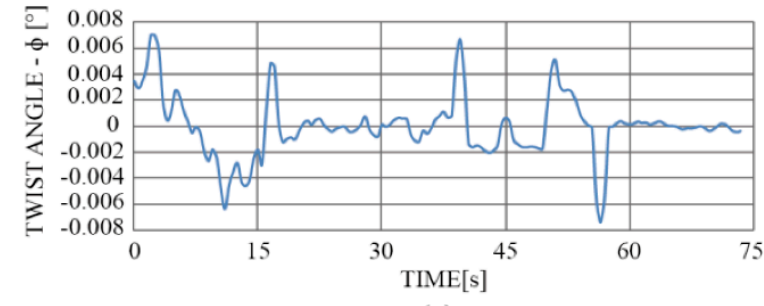

(a)

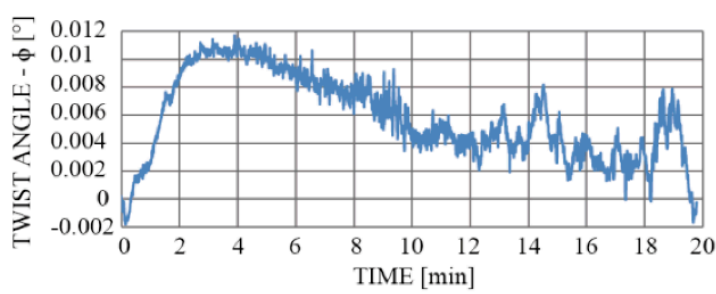

(b)

Figure 5. Influence of the change in (a) Length and (b) Temperature on the angle measurement.

\section{Conclusions}

This paper presented a miniature Fabry Perot twist/rotation sensor with a special asymmetrical microstructure, which allows modulation of the optical path length when the sensor is subjected to twist/rotation. The measured ambiguous range of the sensor was $\pm 90^{\circ}$, at resolution of $0.023^{\circ}$. The sensor was also tested for elongation (extension by $4 \mu \mathrm{m}$ ) and temperature measurements (range between $25^{\circ} \mathrm{C}$ and $125^{\circ} \mathrm{C}$ ), with diminutive influence on the angle measurements, i.e., between $0.012^{\circ}$ and $0.013^{\circ}$.

\section{References}

1. Budinski, V.; Donlagic, D. Fiber-Optic Sensors for Measurements of Torsion, Twist and Rotation: A Review. Sensors 2017, 17, 443, doi:10.3390/s17030443.

2. Cibula, E.; Pevec, S.; Lenardic, B.; Pinet, E.; Donlagic, D. Miniature all-glass robust pressure sensor. Opt. Express 2009, 17, 5098-5106, doi:10.1364/Oe.17.005098.

3. Heo, J.S.; Lee, J.J.; Lim, J.O. A Micro Total Reflective Extrinsic Fabry-Parot Interferometric Fiber Optic Pressure Sensor for Medical Application. Int. J. Mod. Phys. B 2003, 17, 1199-1204, doi:10.1142/S0217979203018740.

4. Pevec, S.; Donlagic, D. All-fiber, long-active-length Fabry-Perot strain sensor. Opt. Express 2011, 19, 15641, doi:10.1364/OE.19.015641.

5. Pevec, S.; Donlagic, D. Miniature all-fiber Fabry-Perot sensor for simultaneous measurement of pressure and temperature. Appl. Opt. 2012, 51, 4536, doi:10.1364/AO.51.004536.

6. Paulicka, I.; Optical fibre Fabry-Perot sensor for the detection of small vibrations and displacements. In Proceedings of the SPIE-The International Society for Optical Engineering, Budapest, Hungary, 9-13 August 1993; pp. 1066-1067. 
7. J. S. Acceta, R. Arrathoon Ultra-sensitive optical sensors for robotic touch and hearing. In Proceedings of the Robotic Intelligence and Productivity Conference, Detroit, MI, USA, 18-19 November 1983; p. 75.

(C)

(C) 2018 by the authors; Licensee MDPI, Basel, Switzerland. This article is an open access article distributed under the terms and conditions of the Creative Commons Attribution (CC BY) license (http://creativecommons.org/licenses/by/4.0/). 